\title{
Pretreatment Tumor DNA Sequencing of KIT and PDGFRA in Endosonography-Guided Biopsies Optimizes the Preoperative Management of Gastrointestinal Stromal Tumors
}

\author{
Per Hedenström ${ }^{1,2}$ (1) $\cdot$ Carola Andersson $^{3} \cdot$ Henrik Sjövall $^{1,2} \cdot$ Fredrik Enlund $^{3} \cdot$ Ola Nilsson $^{3} \cdot$ Bengt Nilsson $^{4}$. \\ Riadh Sadik ${ }^{1}$
}

Published online: 2 March 2020

(c) The Author(s) 2020

\begin{abstract}
Background Neoadjuvant tyrosine kinase inhibitor (TKI) therapy increases the chance of organ-preserving, radical resection in selected patients with gastrointestinal stromal tumors (GISTs). We aimed to evaluate systematic, immediate DNA sequencing of KIT and PDGFRA in pretreatment GIST tissue to guide neoadjuvant TKI therapy and optimize preoperative tumor response. Methods All patients who were candidates for neoadjuvant therapy of a suspected GIST [the study cohort (SC)] were prospectively included from January 2014 to March 2018. Patients were subjected to pretreatment endosonography-guided fine-needle biopsy (EUS-FNB) or transabdominal ultrasound-guided needle biopsy (TUS-NB), followed by immediate tumor DNA sequencing (<2 weeks). A historic (2006-2013) reference cohort (RC) underwent work-up without sequencing before

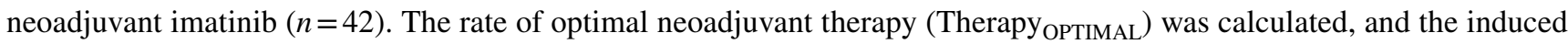
tumor size reduction (Tumor Regression ${ }_{\mathrm{MAX}}, \%$ ) was evaluated by computed tomography (CT) scan.

Results The success rate of pretreatment tumor DNA sequencing in the SC $(n=81)$ was $77 / 81$ (95\%) [EUS-FNB $71 / 74$ (96\%); TUS-NB 6/7 (86\%)], with mutations localized in KIT $(n=58)$, PDGFRA $(n=18)$, or neither gene, wild type $(n=5)$. In patients with a final indication for neoadjuvant therapy, the Therapy OPTIMAL was higher in the SC compared with the RC

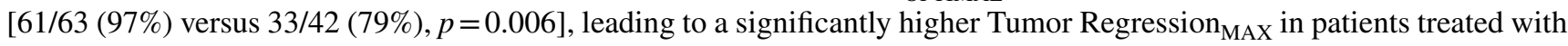
TKI ( $27 \%$ vs. $19 \%, p=0.015)$.

Conclusions Pretreatment endosonography-guided biopsy sampling followed by immediate tumor DNA sequencing of KIT and PDGFRA is highly accurate and valuable in guiding neoadjuvant TKI therapy in GIST. This approach minimizes maltreatment with inappropriate regimens and leads to improved tumor size reduction before surgery.
\end{abstract}

Electronic supplementary material The online version of this article (https://doi.org/10.1007/s40291-020-00451-0) contains supplementary material, which is available to authorized users.

Per Hedenström

per.hedenstrom@vgregion.se

1 Division of Medical Gastroenterology, Department of Internal Medicine, Sahlgrenska University Hospital, Blå Stråket 3, 41335 Gothenburg, Sweden

2 Department of Internal Medicine and Clinical Nutrition, Institute of Medicine, Sahlgrenska Academy, University of Gothenburg, Gothenburg, Sweden

3 Department of Clinical Pathology and Genetics, Sahlgrenska University Hospital, Gothenburg, Sweden

4 Department of Surgery, Sahlgrenska University Hospital, Gothenburg, Sweden

\section{Key Points}

To date, the pretreatment diagnosis and genetic profiling of tumors has been challenging and imperfect in patients with suspected gastrointestinal stromal tumors (GISTs).

In the current work, we show that endosonographyguided fine-needle biopsy sampling followed by nextgeneration sequencing of KIT and PDGFRA is highly accurate for the diagnosis and mutational analysis of GISTs already at an early, pretreatment stage.

The suggested work-up enables neoadjuvant, tyrosine kinase inhibitor therapy firmly based on tumor genomics data. Moreover, we demonstrate that the suggested approach leads to a significantly lower number of patients being maltreated with suboptimal neoadjuvant therapy regimens and results in a significantly greater tumor size reduction before surgery. 


\section{Introduction}

In modern cancer care, it is often as important as it is challenging to initiate an effective treatment with limited toxicity. This statement is certainly valid regarding gastrointestinal stromal tumors (GISTs). The anti-tumoral effect of targeted therapy with tyrosine kinase inhibitors (TKIs) can be dramatic in GISTs $[1,2]$, but TKIs, such as imatinib and sunitinib, are also associated with substantial side effects [3-5].

Patient survival has improved significantly thanks to imatinib therapy, both in patients suffering from metastatic GISTs [3, 4, 6] and in resected patients (adjuvant therapy) with tumors larger than $3 \mathrm{~cm}$ [5]. Neoadjuvant imatinib for preoperative, tumor downsizing induces rapid tumor cell apoptosis [7], facilitates surgical, radical resection [8-10], and leads to a high disease-specific survival [11] without an increased post-operative complication rate [7, 12].

However, the individual tumor response to imatinib therapy is intimately related to the tumor genetic profile [13]. A majority of sporadic GISTs occur due to primary mutations in either the KIT or PDGFRA genes. Some genetic subgroups, such as the common $K I T$ exon 11 mutants and the rare $P D G F R A$ exon 12 mutants, respond well to standard dose imatinib (400 mg daily) [14-16] (Table 1). A rare exception is the KIT exon 11 p.(L576P) variant, which is far less sensitive [17]. An intermediate response is seen in KIT exon 9 mutants requiring high-dose imatinib $(800 \mathrm{mg}$ daily) $[6,15,18]$. A few other mutations, such as the $P D G$ $F R A$ exon 18 p.(D842V) mutant, are completely resistant to imatinib [16, 19] (Table 1). Sporadic tumors without any detected mutation in KIT or PDGFRA are called wild type (WT) tumors and are regarded as nonsensitive to imatinib therapy [20]. Instead, such patients are candidates for upfront surgery or clinical trials evaluating alternative therapies [21].

Therefore, the adequacy and effect of neoadjuvant imatinib therapy is dependent on correct information regarding the precise mutation in each individual tumor. Admittedly, PDGFRA mutants are most commonly found in the stomach and KIT exon 9 mutants in the small intestine, but the tumor origin alone cannot predict the genetic profile [13]. Likewise, the epithelioid cell type is common among PDGFRA mutants, but the morphology of the tumor cells per se does not sufficiently reveal the underlying mutation [22].

The pretreatment diagnosis and genetic profiling of suspected GISTs have been limited by imperfect available diagnostic techniques. Performing a transabdominal ultrasound-guided needle biopsy (TUS-NB) requires an accessible tumor of a reasonably large size. Routine endoscopy with forceps biopsy and endoscopic ultrasound with fineneedle aspiration for cytology (EUS-FNA) both have a low diagnostic accuracy [23, 24]. However, the use of a new generation of EUS needles aimed for histology [endosonography-guided fine-needle biopsy (EUS-FNB)] can ameliorate the shortcomings of other diagnostic approaches and lead to early genetic profiling of GISTs [25].

In summary, the effective, neoadjuvant therapy in GISTs is dependent on accurate diagnostic information including tumor genomic data. The overall goal of this work was to evaluate EUS-FNB and TUS-NB followed by immediate mutational analysis of KIT and PDGFRA in pretreatment tumor tissue for the early detection of unfavorable mutations with respect to imatinib therapy. An additional aim was to analyze the clinical impact, especially the efficacy of treatment, of such a standardized work-up in patients considered for neoadjuvant therapy.

\section{Methods}

\subsection{Study Setting and Study Subjects}

The Sahlgrenska University Hospital (SUH) is a tertiary center for EUS and for the work-up and care of all patients with GISTs in the region of West Sweden (population approximately 1.9 million). All incident GIST cases are managed via a preoperative, multidisciplinary therapy conference (MDTC).

All patients $>18$ years referred to the SUH for the preoperative diagnostic work-up of a suspected GIST during the period January 2014-March 2018, hereafter called the study cohort (SC), were eligible for inclusion in this prospective, singlecenter study. Patients with probable GIST but with a condition requiring urgent surgery, such as ileus or tumor bleeding, were not eligible for study inclusion. Eligible patients were excluded if the diagnostic work-up resulted in a final diagnosis other than GIST.

The patients with GIST advocated for and treated with neoadjuvant therapy in the same center during the period 2006-2013 constituted the reference population-hereafter called the reference cohort (RC).

This study was approved by the Regional Ethical Review Board of Gothenburg. Written informed consent was obtained from all the study participants. The study was registered at ClinicalTrials.gov (NCT02360839).

\subsection{Tumor Sampling Procedures}

\subsubsection{Study Cohort}

The work-up strategy of all referrals was determined by the study surgeon (BN). Primarily, the study participants were subjected to high-priority, pretreatment EUS-FNB. In tumors considered out of reach by oral access EUS, 
Table 1 Anti-tumoral effect of standard dose imatinib in GISTs in relation to the primary mutation of the tumor

\begin{tabular}{|c|c|c|c|}
\hline \multirow[t]{2}{*}{ Tumor genomic profile } & \multicolumn{3}{|c|}{ Anti-tumoral effect of standard dose IMA ${ }^{\mathrm{a}}$} \\
\hline & Adequate & Reduced & Poor \\
\hline \multicolumn{4}{|l|}{ Favorable mutations } \\
\hline KIT exon 11 [mutants except p.(L576P)] $[14,15]$ & $\mathrm{X}$ & & \\
\hline KIT exon 13 [14] & $\mathrm{X}$ & & \\
\hline PDGFRA exon $12[14,15]$ & $\mathrm{X}$ & & \\
\hline PDGFRA exon $14[16]$ & $\mathrm{X}$ & & \\
\hline PDGFRA exon 18[mutants except p.(D842V)] [28] & $\mathrm{X}$ & & \\
\hline \multicolumn{4}{|l|}{ Unfavorable mutations } \\
\hline KIT exon $9[18]$ & & $\mathrm{X}$ & \\
\hline Wild type $[15,48]$ & & $\mathrm{X}$ & \\
\hline KIT exon 11 p.(L576P) [17] & & & $\mathrm{X}$ \\
\hline KIT exon $17[28,29]$ & & & $\mathrm{X}$ \\
\hline PDGFRA exon 18 p.(D842V) $[16,19,28]$ & & & $X$ \\
\hline
\end{tabular}

GIST gastrointestinal stromal tumor, IMA imatinib

${ }^{\text {a }}$ Standard dose IMA $=400 \mathrm{mg}$ daily

high-priority TUS-NB was performed (Fig. 1). Any EUSFNB was performed by one of the study endosonographers (RS/PH) using a linear echoendoscope (EG3870UTK, Pentax, Tokyo, Japan) and a 22-gauge biopsy needle (Procore $^{\mathrm{TM}}$, Wilson-Cook Medical, Limerick, Ireland or Sharkcore ${ }^{\mathrm{TM}}$, Medtronic, Minneapolis, USA). Any TUSNB were performed at the radiology department using an 18-gauge biopsy needle (Bard Biopsy Systems, Tempe, USA). All samples were sent to the study pathologist $(\mathrm{ON})$.

\subsubsection{Reference Cohort}

During the RC time frame (2006-2013), EUS constituted one diagnostic option, but the method was not systematically performed before MDTC. If EUS was conducted, sampling was mainly performed by the contemporary standard technique, i.e. EUS-FNA for cytology (22/25gauge Echotip ${ }^{\mathrm{TM}}$, Wilson-Cook Medical). If TUS-NB was conducted, sampling was performed as above.

\subsection{Tumor DNA Sequencing}

In the SC, the samples of EUS-FNB and TUS-NB were subjected to tumor DNA sequencing immediately after the histopathology assessment (<1 week). During 2014-2015, and for research purposes, the corresponding surgical specimens of all of the resected cases were also subjected to tumor DNA sequencing to confirm the detected mutations [25]. During 2016-2018, this procedure was performed only in cases with a WT profile identified in the pretreatment sample (to confirm the absence of a mutation) and in cases with failed pretreatment sequencing.

In the RC, mostly due to the lack of appropriate pretreatment tumor samples, no sequencing of KIT and PDGFRA was performed within a reasonable time to enable genotypeguided management at the MDTC. Sequencing of the surgical specimens or of available preoperative work-up samples was instead performed at a later stage.

Before sequencing (SC and $\mathrm{RC}$ ), the approximate number of tumor cells in each sample was estimated. Then, the tumor area was manually microdissected from the formalinfixed paraffin-embedded (FFPE) tissue. Five micrometer thick sections from each sample were cut and put into a 1.5$\mathrm{ml}$ tube. DNA was isolated using the QIAamp DNA FFPE tissue KIT (Qiagen $\mathrm{GmbH}$, Hilden, Germany), following the manufacturer's instructions. The DNA concentration was determined using a Qubit 2.0 Fluorometer (Life Technologies, Carlsbad, CA, USA).

\subsubsection{Sanger Sequencing}

Between January 2014 and December 2015, Sanger sequencing was the method used in all cases. For the purpose of sequencing, $200 \mathrm{ng}$ of DNA was used to detect mutations in KIT exons 9, 11, and 13 and PDGFRA exons 12 and 18 with primers designed in-house (details available on request) and the Multiplex PCR KIT (Qiagen) according to the manufacturer's instructions. Sanger sequencing of the amplicons was performed with both the forward and reverse primers using the BigDye ${ }^{\mathrm{TM}}$ Terminator v1.1 Cycle Sequencing KIT 


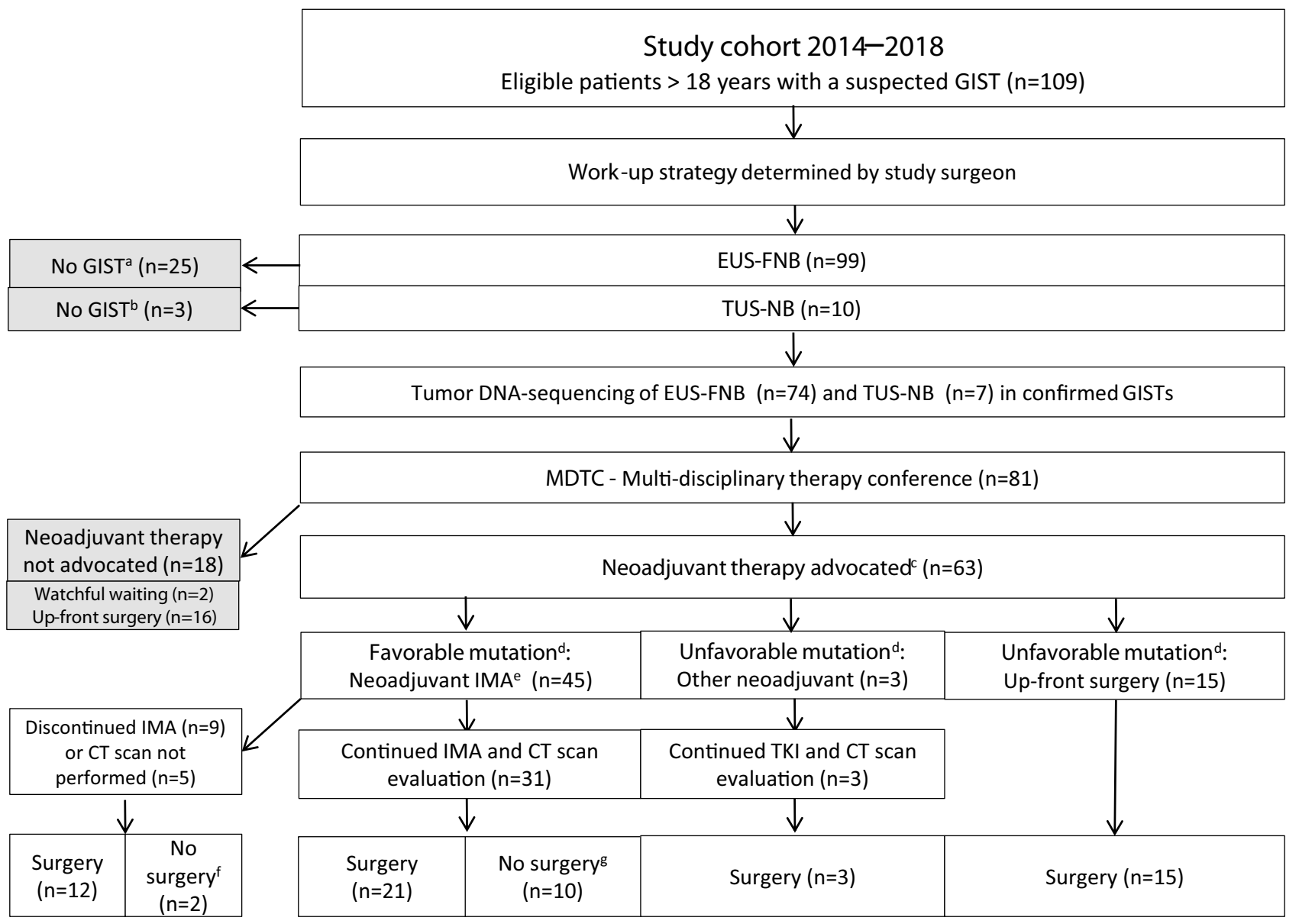

Fig. 1 Flow chart of patients with a suspected GIST eligible for study enrollment January 2014-March 2018 in Sahlgrenska University Hospital. ${ }^{a}$ Diagnostic entities in non-GIST lesions subjected to EUS-FNB: leiomyoma $(n=10)$; schwannoma $(n=5)$; adenocarcinoma $(n=3)$; leiomyosarcoma $(n=2)$; epithelioid sarcoma $(n=1)$; benign fibrinoid tumor $(n=1)$; ganglioneurinoma $(n=1)$; lipoma $(n=1)$; malignant peripheral nerve sheath tumor $(n=1)$. ${ }^{\mathrm{b}}$ Diagnostic entities in non-GIST lesions subjected to TUS-NB: desmoid tumor $(n=1)$; adenocarcinoma $(n=1)$; leiomyosarcoma $(n=1)$. ${ }^{\mathrm{c} C r i t e r i a}$ for the initiation of down-sizing therapy according to the main body

with the ABI PRISM ${ }^{\mathrm{TM}}$ 3130xl Genetic Analyzer (Applied Biosystems, Foster City, CA, USA).

\subsubsection{Next-Generation Sequencing}

From January 2016 onwards, next-generation sequencing (NGS) was the method used in all cases. For the purpose of sequencing, $10 \mathrm{ng}$ of DNA was used to prepare barcoded libraries with the Ion AmpliSeq ${ }^{\mathrm{TM}}$ Library KIT 2.0 (Life Technologies). The Cancer Hotspot Panel v2 (Life Technologies) covering 207 targeted regions in 50 cancer-related genes (Supplementary Table 1 in the Electronic Supplementary Material) was used to identify mutations in KIT (exons 9-11, 13-15, and 17-18), PDGFRA (exons 12, 14, 15, and of the manuscript. ${ }^{\mathrm{d}}$ According to Table $1 .{ }^{\mathrm{e}}$ Neoadjuvant therapy with imatinib mesylate in standard dose (400 mg daily). ${ }^{\mathrm{f}}$ The two patients were considered unfit for the extensive surgery needed. Instead, the patients were provided the best palliative treatment. ${ }^{\mathrm{g}}$ The reason for surgery not being performed at the end of the study period among the ten patients was: (a) patients awaiting surgery, $n=1$; (b) patients with concomitant malignancy being unfit for surgery, $n=1$; (c) patients with a remaining large tumor burden not yet considered eligible for radical resection, $n=4$; (d) patients with advanced comorbidity being unfit for surgery, $n=3$

18), and $B R A F$ (exon 15). Template preparation and enrichment were performed with the IonChef (Life Technologies). Eight barcoded samples were pooled per Ion $318^{\mathrm{TM}}$ chip and sequenced on the Ion PGM ${ }^{\mathrm{TM}}$ System (Life Technologies). All steps were performed according to the manufacturer's instructions.

Alignment to the hg19 human reference genome and variant calling were performed by the Torrent Suite Software v5.2.2 (Life Technologies). To identify hotspot variants, the Variant Caller v5.2.0.34 (Life Technologies) was used. Alignment was visually inspected with the Integrative Genomics Viewer (IGV) modified by IonTorrent v5.01 (0) (Broad Institute, Cambridge, MA, USA). 


\subsection{The MDTC and Neoadjuvant Therapy}

The results of pathology and pretreatment sequencing with mutational analysis were presented to the Sahlgrenska MDTC for decisions on further preoperative management. The SUH multidisciplinary GIST team advocates neoadjuvant TKI therapy in all patients with GISTs (without advanced comorbidity) if the tumor diameter $>3 \mathrm{~cm}$ and/ or if the tumor is borderline resectable, e.g., situated in the gastroesophageal junction or in the duodenum. The SUH experience (nonpublished data) is that the use of neoadjuvant therapy in relatively small tumors $(3-5 \mathrm{~cm})$ facilitates surgery and minimizes the perioperative complication rate. The cases not advocated for neoadjuvant therapy were managed by upfront resection or watchful waiting (Fig. 1), and these cases were not further analyzed within the study.

In the SC patients, standard dose imatinib (400 mg daily) was primarily initiated by the MDTC, but only if the pretreatment mutation detected was favorable and indicated full sensitivity to imatinib (Table 1). If the mutation detected was unfavorable and indicated imatinib resistance or reduced sensitivity to imatinib, an alternative downsizing strategy was chosen or upfront surgery was performed (Table 1 and Fig. 1). Inexorably, neoadjuvant therapy will result in a low mitotic index in surgical specimens of GISTs with favorable mutations. Therefore, adjuvant therapy after surgery was more liberally advocated by the MDTC in patients having tumors with an indeterminate risk.

In the RC patients, standard dose imatinib (400 mg daily) was initiated in all cases advocated for neoadjuvant therapy, as no data on the tumor mutation profiles were or could be provided to the MDTC before decisions were made on further management and therapy.

\subsection{Follow-Up and Surgery}

After the MDTC, the study subjects were monitored by regular visits to the outpatient unit. Neoadjuvant therapy was continued for a minimum of 3 months and a maximum of 15 months. Therapy was stopped and surgery was attempted either when the estimated maximal tumor size reduction was obtained or when the tumor size reduction was high enough to enable organ-saving radical resection.

Any side effects of neoadjuvant therapy were evaluated and recorded according to the National Cancer Institute Common Terminology Criteria for Adverse Events (CTCAE) system [26] ranging from mild to life threatening (grade 1: mild; grade 2: moderate; grade 3: severe; grade 4: life-threatening or disabling; grade 5: death related to side effects). Mild side effects were usually treated by symptomatic therapy. In patients experiencing continuous, moderate-severe side effects (and with the intent to avoid complete therapy discontinuation), the dosage of imatinib was reduced to $100-200 \mathrm{mg}$ daily. If imatinib dose reduction did not lead to reduced side effects, neoadjuvant therapy was discontinued.

As in other studies analyzing the value of neoadjuvant imatinib therapy [11,12], repeated computed tomography (CT) scans and the application of the RECIST criteria [27] were used for tumor response evaluation. The tumor size reduction, Tumor Regression ${ }_{\mathrm{MAX}},(\%)$ was calculated by comparing the maximum cross-sectional tumor size $(\mathrm{mm})$ of the pretreatment CT scan (Pre-CT $\mathrm{C}_{\max }$ ) with the followup CT scan (Post-CT ${ }_{\max }$ ) performed 12-16 weeks after the initiation of neoadjuvant TKI therapy:

Tumor Regression $_{\mathrm{MAX}}(\%)=100$

$$
\times\left(\text { Pre }-\mathrm{CT}_{\max }-\text { Post }-\mathrm{CT}_{\max }\right) / \text { Pre }-\mathrm{CT}_{\max } \text {. }
$$

Patients in any cohort were excluded from the Tumor Regression $_{\text {MAX }}$ analysis if they discontinued therapy before 12 weeks of treatment or an evaluation CT scan for some reason was not performed at the appropriate time. Based on the tumor characteristics and the tumor response to therapy, the appropriate time for resection was determined by the study surgeon (BN).

Finally, each case was categorized either as subjected to optimal neoadjuvant therapy (OT) or non-optimal neoadjuvant therapy (NOT) based on the detected genomic status, the therapy initiated, and according to Table 1 [14-20, 28, 29]. The rate of optimal neoadjuvant therapy, Therapy ${ }_{\text {OPTIMAL }},(\%)$ in each cohort (SC and RC) was calculated:

Therapy $_{\text {OPTIMAL }}=100 \times n_{\mathrm{OT}} /\left(n_{\mathrm{OT}}+n_{\mathrm{NOT}}\right)$.

\subsection{Outcomes}

The primary outcome was the success rate $(\%)$ of pretreatment sequencing using the EUS-FNB/TUS-NB samples in an intention-to-diagnose analysis. Success was defined as a conclusive mutational analysis as assessed by the study geneticist (FE) and accomplished in time for the MDTC ( $<2$ weeks after sampling). In tumors classified as WT in pretreatment samples, confirmation of the WT profile was also required by sequencing of the corresponding resection specimens.

The secondary outcomes were (1) the Therapy OPTIMAL (\%), (2) the preoperative tumor size reduction, Tumor Regression $_{\text {MAX }}(\%)$, (3) the R0-resection rate, and (4) short-term parameters related to surgical resection. 


\subsection{Statistics}

Descriptive, pretreatment baseline data of the study subjects and the tumors were expressed as the median and the interquartile range (IQR). The NIH classification was not, and could not, be used to calculate the prognostic risk in surgical specimens as neoadjuvant therapy affects both the tumor size and the mitotic rate. Instead, imaging data and pretreatment tumor samples were used to calculate the tumor size and the tumor proliferation rate (Ki-67 index).

Fisher's exact test and Student's $t$ test (Tumor Regression $_{\text {MAX }}$ ) were used to test for significant differences in the secondary outcome variables comparing the $\mathrm{SC}$ and the RC. The $95 \%$ confidence interval (CI) was calculated when appropriate. All tests were two-tailed and conducted at a statistical significance level of $p<0.05$. The software SPSS Statistics version 22.0 (IBM Corp., Chicago, IL, USA) was used for all calculations.

\section{Results}

Of 109 eligible patients, 81 study participants with a final diagnosis of GIST were included in the SC, of which 63 participants were advocated neoadjuvant therapy (Fig. 1, Table 2, and Supplementary Table 2).

All cases $(n=81)$ subjected to EUS-FNB $(n=74)$ and TUS-NB $(n=7)$ received a preoperative diagnosis of GIST including conclusive immunohistochemistry. In one case only, a repeated EUS-FNB procedure was required due to a nonconclusive index EUS. One adverse event was recorded [adverse event rate: TUS-NB 0/7 (0\%); EUSFNB 1/74 (1.4\%)]. The event was local bleeding post-EUS from a GIST situated in the stomach. The bleeding was stopped via gastroscopy by the injection of epinephrine in the tumor. No case was lost from follow-up.

The RC consisted of 42 patients (Table 2).

\subsection{The Primary Outcome}

In the SC, the success rate of pretreatment sequencing was $77 / 81(95 \%)$ of all cases counted (Table 3 ) and 61/63 (95\%) in the cases advocated for neoadjuvant therapy. There was no significant difference in success rate comparing Sanger sequencing with NGS (Table 3). The distribution of the detected mutations was according to Table 2 and Supplementary Table 1, with no primary mutations detected in less frequently mutated genes such as the $B R A F$ gene.

\subsubsection{Sequencing of the EUS-FNB Samples}

The success rate of pretreatment sequencing of the EUSFNB samples was 71/74 (96\%) [Sanger 30/31 (97\%); NGS $41 / 43$ (95\%), $p=1.0$, Table 3]. In the first unsuccessful case (a 2-cm gastric GIST situated near the gastroesophageal junction), the amount of EUS-FNB tissue was insufficient for the analysis. Sequencing of the corresponding surgical specimen showed a KIT exon 11 mutation. In the second (a 3 -cm duodenal GIST) and the third (a 22-cm gastric GIST) unsuccessful cases, the pretreatment sequencing showed WT profiles, while the post-operative specimen sequencing detected a KIT exon 11 mutation in both cases. In both the first and the second case, the indication for neoadjuvant therapy was not the tumor size, but rather the tumor position and the possibility of avoiding extensive surgery after tumor downsizing. In 21 cases, sequencing of the corresponding surgical specimen confirmed the primary mutation detected in the pretreatment EUS-FNB sample.

\subsubsection{Sequencing of TUS-NB Samples}

The success rate of pretreatment sequencing of the TUS-NB samples was 6/7 (86\%) [Sanger 3/4 (75\%); NGS 3/3 (100\%), $p=1.0$, Table 3]. In three cases, sequencing of the corresponding surgical specimen confirmed the primary mutation detected in the pretreatment TUS-NB sample. In one of these three cases, a patient treated with neoadjuvant imatinib, there was a secondary mutation in the BRAF gene detected by the NGS in the surgical specimen. The primary mutation of this case was KIT exon 11 p.Q556_N564delinsH.

\subsection{The Secondary Outcomes}

\subsubsection{Rate of Optimal Neoadjuvant Therapy}

A favorable mutation was detected in $45 / 63$ (71\%) of the SC tumors advocated for neoadjuvant therapy (Fig. 1 and Table 4). In these patients, a standard dosage of imatinib $400 \mathrm{mg}$ daily was initiated.

The rate of optimal neoadjuvant therapy (Therapy OPTIMAL $_{\text {) }}$ was significantly higher in the SC compared with the RC [61/63 (97\%) vs. 33/42 (79\%), $p=0.006$, Table 4].

\subsubsection{Tumor Size Reduction}

In total, 90 patients were initiated on neoadjuvant TKI therapy [SC $n=48$ (imatinib $n=45$, sunitinib $n=3$ ) (Fig. 1); RC $n=42$ (imatinib $n=42$ )].

Among the 48 SC patients, at least one moderate side effect related to neoadjuvant TKI therapy was recorded in 14 patients (29\%) (all treated with imatinib). The discontinuation of imatinib therapy due to side effects was recorded in 
Table 2 The baseline characteristics of the study cohort and the reference cohort

\begin{tabular}{|c|c|c|c|c|}
\hline & \multicolumn{3}{|l|}{ Study cohort } & \multirow[t]{2}{*}{ Reference cohort } \\
\hline & All cases & Neoadjuvant advocated & $\begin{array}{l}\text { Neoadjuvant not } \\
\text { advocated }\end{array}$ & \\
\hline \multicolumn{5}{|l|}{ Patient characteristics } \\
\hline Number of patients, $n$ & 81 & 63 & 18 & 42 \\
\hline Patient age, median (IQR) & $69(62-74)$ & $69(64-75)$ & $65(57-73)$ & $66(60-74)$ \\
\hline Patient gender, M/F & $39 / 42$ & $32 / 31$ & $7 / 11$ & $25 / 17$ \\
\hline \multicolumn{5}{|l|}{ Tumor characteristics (pretreatment) } \\
\hline \multicolumn{5}{|l|}{ Tumor origin, $n$} \\
\hline Esophagus & 1 & 1 & 0 & 0 \\
\hline Stomach (fundus/body/antrum) & $71(24 / 40 / 7)$ & $55(18 / 33 / 4)$ & $16(6 / 7 / 3)$ & $31(16 / 14 / 1)$ \\
\hline Small bowel (duod/jejun/ileum) & $9(7 / 0 / 2)$ & $7(5 / 0 / 2)$ & $2(2 / 0 / 0)$ & $11(4 / 3 / 4)$ \\
\hline Tumor size ${ }^{\mathrm{a}}, \mathrm{mm}$, median (IQR) & $45(29-75)$ & $52(40-90)$ & $24(20-28)$ & $90(49-163)$ \\
\hline $\begin{array}{l}\text { Tumor proliferation rate }{ }^{\mathrm{b}} \text { (Ki-67 index), } \\
\text { median (IQR) }\end{array}$ & $4.0(2.0-6.0)$ & $4.0(2.0-6.0)$ & $3.3(2.2-4.0)$ & $7.0(2.6-11.8)$ \\
\hline \multicolumn{5}{|l|}{ Tumor genomic profile (gene/exon) } \\
\hline$K I T$ exon 9 & 2 & 2 & 0 & 1 \\
\hline$K I T$ exon $11^{\mathrm{c}}$ & 52 & 39 & 13 & 34 \\
\hline$K I T$ exon $13^{\mathrm{d}}$ & 3 & 3 & 0 & 1 \\
\hline$K I T$ exon 17 & 1 & 1 & 0 & 0 \\
\hline PDGFRA exon 12 & 3 & 2 & 1 & 0 \\
\hline PDGFRA exon 14 & 1 & 0 & 1 & 0 \\
\hline PDGFRA exon 18 p. (D842V) & 12 & 10 & 2 & 5 \\
\hline PDGFRA exon 18 non-p. (D842V) & 2 & 2 & 0 & 0 \\
\hline Wild type & 5 & 4 & 1 & 1 \\
\hline
\end{tabular}

$F$ female, $I Q R$ interquartile range, $M$ male

${ }^{\text {a }}$ Pretreatment tumor size

${ }^{\mathrm{b}}$ The Ki-67 index was assessed and calculated in the available and adequate pretreatment samples with no recorded statistical difference in the Ki-67 index comparing tumors in various locations (esophagus vs. stomach vs. duodenum) and tumors of various genomic status (KIT mutation vs. PDGFRA mutation vs. WT profile)

${ }^{\mathrm{c}}$ Details on the KIT exon 11 mutations are provided in Supplementary Table 1 (see the electronic supplementary material)

${ }^{\mathrm{d}}$ All mutants were p. (K642E)

Table 3 The primary outcome: the success rate of pretreatment tumor DNA-sequencing

\begin{tabular}{|c|c|c|c|c|}
\hline \multirow[t]{2}{*}{ Sample type } & \multicolumn{2}{|c|}{ Sequencing modality } & \multirow[t]{2}{*}{$P$ value } & \multirow[t]{2}{*}{ Total } \\
\hline & Sanger & NGS & & \\
\hline $\begin{array}{c}\text { EUS-FNB } \\
(n=74)\end{array}$ & $30 / 31(97 \%)$ & $41 / 43(95 \%)$ & 1.0 & $71 / 74(96 \%)$ \\
\hline TUS-NB $(n=7)$ & $3 / 4(75 \%)$ & $3 / 3(100 \%)$ & 1.0 & $6 / 7(86 \%)$ \\
\hline $\begin{array}{l}\text { All samples } \\
(n=81)\end{array}$ & $33 / 35(94 \%)$ & $44 / 46(96 \%)$ & 1.0 & $77 / 81(95 \%)$ \\
\hline
\end{tabular}

$E U S-F N B$ endosonography-guided fine-needle biopsy, NGS nextgeneration sequencing, TUS-NB transabdominal ultrasound-guided needle biopsy nine out of 45 patients (20\%) (edema $n=4$; dermatitis $n=2$; nausea $n=2$; diarrhea $n=1$, Fig. 1 ).

Among the $42 \mathrm{RC}$ patients, at least one moderate side effect related to neoadjuvant TKI therapy was recorded in ten patients $(24 \%)$. The discontinuation of imatinib therapy due to side effects was recorded in six out of 42 patients (14\%) (edema $n=3$; dermatitis $n=2$; nausea $n=1$ ).

Finally, 66 out of 90 patients (73\%) [SC $n=34 / 48$ (imatinib $n=31$; sunitinib $n=3$ ); RC $n=32 / 42$ (imatinib $n=32)$ ] completed neoadjuvant TKI and had at least one follow-up CT scan performed (Fig. 1). The mean tumor size

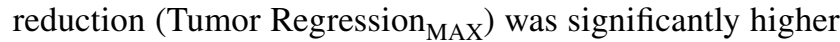
in the SC compared with the RC [27\% (95\% CI 22-31) 
vs. 19\% (95\% CI 14-24), $p=0.015$, Fig. 2a]. The Tumor Regression $_{\mathrm{MAX}}$ results in relation to the tumor genomic profile of individual cases are shown in Figs. $2 b$ and c. The mean Tumor Regression ${ }_{\text {MAX }}$ in cases with exclusively favorable mutations was comparable in the SC and the RC [27\% (95\% CI $23-31$ ) vs. $24 \%$ (95\% CI 21-27), $p=0.27$ ].

\subsubsection{R0-Resection Rate and Other Parameters Related to Surgical Resection}

Among the cases completing neoadjuvant TK therapy and finally proceeding to surgical resection (SC $n=29, \mathrm{RC}$ $n=28$, Fig. 1), the R0-resection rate was not significantly higher comparing the SC $(24 / 29,83 \%)$ and the RC $(24 / 28$,
$86 \%, p=1.0)$. The mean size of the resection specimens in the SC and the RC was $64 \mathrm{~mm}(95 \% \mathrm{CI} 46-80)$ and $78 \mathrm{~mm}$ (95\% CI 58-98), respectively. Other short-term outcome parameters related to surgery are presented in Table 5. The mean duration of neoadjuvant therapy before surgery did not differ between the two cohorts [SC 8.2 months (95\% CI $6.2-10.2$ ) vs. RC 8.3 months (95\% CI 6.0-10.6), $p=0.94]$.

\section{Discussion}

In this prospective study on consecutive patients, we showed that the rapid and accurate pretreatment genomic profiling of GISTs can be achieved in a vast majority of cases. The early

Table 4 The secondary outcome: the rate of optimal neoadjuvant therapy (Therapy ${ }_{\text {OPTIMAL }}$ )

\begin{tabular}{|c|c|c|c|}
\hline & Study cohort & Reference cohort & $P$ value \\
\hline \multirow[t]{2}{*}{ Therapy } & $61 / 63(97 \%)$ & $33 / 42(79 \%)$ & 0.006 \\
\hline & IMA standard $^{\mathrm{a}}$ & Alternative $\mathrm{TKI}^{\mathrm{b}}$ & Other therapy $^{\mathrm{d}}$ \\
\hline \multicolumn{4}{|l|}{ Study cohort } \\
\hline \multicolumn{4}{|l|}{ Favorable mutations } \\
\hline$K I T$ exon $11^{\mathrm{e}}$ & 37 & & $\mathbf{1}^{\mathrm{h}}$ \\
\hline KIT exon 13 & 3 & & \\
\hline PDGFRA exon 12 & 2 & & \\
\hline$P D G F R A$ exon $18^{\mathrm{f}}$ & 2 & & \\
\hline \multicolumn{4}{|l|}{ Unfavorable mutations } \\
\hline KIT exon 9 & $1^{g}$ & 1 & \\
\hline Wild type & & 2 & 2 \\
\hline KIT exon 11 p. (L576P) & & & 1 \\
\hline KIT exon 17 p. (Y823D) & & & 1 \\
\hline PDGFRA exon 18 p. (D842V) & & & 10 \\
\hline \multicolumn{4}{|l|}{ Reference cohort } \\
\hline \multicolumn{4}{|l|}{ Favorable mutations } \\
\hline$K I T$ exon $11^{\mathrm{e}}$ & 32 & & \\
\hline KIT exon 13 & 1 & & \\
\hline \multicolumn{4}{|l|}{ Unfavorable mutations } \\
\hline KIT exon 9 & 1 & & \\
\hline Wild type & 1 & & \\
\hline KIT exon 11 p. (L576P) & 2 & & \\
\hline PDGFRA exon $18 \mathrm{p} .(\mathrm{D} 842 \mathrm{~V})$ & 5 & & \\
\hline
\end{tabular}

The number of cases subjected to the different types of neoadjuvant therapy and with respect to the tumor's genetic profile. Numbers in bold signify the cases subjected to non-optimal neoadjuvant therapy according to Table

IMA imatinib, TKI tyrosine kinase inhibitor, WT wild type

${ }^{a}$ IMA standard dose (400 mg daily)

${ }^{b}$ IMA high dose (800 mg daily) or other TKI therapy besides IMA, i.e., sunitinib

${ }^{\mathrm{d}}$ Non-TKI based therapy, such as regular chemotherapy or upfront surgery

${ }^{\mathrm{e}}$ Includes all variants of mutations in KIT exon 11 except p. (L576P)

${ }^{\mathrm{f}}$ All variants of mutations in PDGFRA exon 18 except $\mathrm{p}$. (D842V)

${ }^{g}$ Pretreatment mutational analysis failed, resulting in the incorrect regimen of standard dose IMA in a KIT exon 9 mutant

${ }^{\text {h}}$ Pretreatment mutational analysis falsely showed a WT profile, resulting in upfront surgery of a KIT exon 11 mutant 
mutational analysis of the tumors had an important impact on the preoperative management of patients, demonstrated by only a few cases subjected to inadequate neoadjuvant therapy. Consequently, first-line, standard dose imatinib was initiated only in patients with a favorable tumor mutation, leading to a maximized chance of effective, preoperative tumor size reduction. Primarily, the results were enabled via the combination of high-priority EUS-guided biopsy sampling followed by immediate sequencing of KIT and PDGFRA in the acquired tumor tissue.

Without tumor genomic information, the prediction of the efficacy of neoadjuvant TKI therapy in GISTs will be more or less a qualified guess. Obviously, some tumors will respond to standard dose imatinib by chance $[10,12,30]$. However, there is a substantial risk of initiating a suboptimal regimen and delaying alternative strategies or surgery, as in patients with WT tumors or PDGFRA exon 18 p.(D842V) tumors. In the presented work, we could define the subset of patients eligible for standard dose imatinib in all but one patient. Importantly, 18 patients advocated for neoadjuvant therapy had a tumor mutation associated with nil or reduced imatinib sensitivity. In 17 of these patients, the mutation was detected before therapy was initiated, which led to a modified treatment plan.

The toxicity profile of imatinib is another argument for pretreatment mutational analysis. The frequency of therapy discontinuation reached as high as $35 \%$ in a study using $600 \mathrm{mg}$ imatinib daily for neoadjuvant treatment [12]. High-dose $800 \mathrm{mg}$ imatinib is associated with even more toxicity [3, 4] and should be initiated only when genetically motivated, such as in KIT exon 9 mutants. In a relatively recent publication by Kurokawa and coworkers, daily $400 \mathrm{mg}$ imatinib was used for neoadjuvant therapy as in our study [10]. The authors recorded a need for imatinib dose reduction in 14 out of 53 patients $(26 \%)$ and a final need for imatinib discontinuation in seven out of 53 patients (13\%). Even though we recorded a somewhat higher need for imatinib discontinuation in the SC, but not in the RC, these numbers are comparable to the data reported by Kurokawa et al. [10] and lower than the rate of discontinuation reported by Wang et al. [12].

By comparing the SC with a historic cohort of comparable GIST patients, we could demonstrate that the mean tumor size reduction induced by neoadjuvant therapy was significantly higher if pretreatment mutational analysis of GIST was performed to guide therapy. That finding is important as the radiographic tumor response may affect the chance of radical resection (R0) and subsequently the risk of tumor recurrence, at least in patients with advanced GISTs $[9,11]$. In the presented work, we did not record any increased frequency of R0 resections in the SC compared with the RC not subjected to pretreatment sequencing. To prove such a hypothesis, a high number of patients in two risk-matched study populations would be required, which is beyond the capacity of a single center. Nevertheless, sequencing followed by tailored, genotype-driven therapy will most likely lead to the reduction of suboptimal TKI regimens, i.e., unnecessary switches of TKIs, and thereby reduce the time to an adequate anti-tumoral effect. We recorded a numerically lower frequency of post-operative complications in the SC compared with the RC (Table 5). It can be hypothesized, though definitely not proven, that pretreatment sequencing followed by adequate neoadjuvant therapy to some extent contributed to this result. It would be interesting if future studies could address this topic.

Others have evaluated the effect of neoadjuvant imatinib in prospective studies of GIST [7, 10, 12, 30-32]. In addition, our group has explored the feasibility of mutational analysis in pretreatment EUS-FNB, in a majority of study subjects late after diagnosis [25]. However, to the best of our knowledge, the presented, prospective work is the first study performing systematic pretreatment sequencing of GIST tissue in a high number of consecutive patients to guide neoadjuvant therapy.

There are other potential advantages of pretreatment sequencing of GISTs. First, some GISTs $(<5 \%)$ are c-KITnegative in immunostaining [33]. Certainly, complementary immunostaining with DOG-1 has a high sensitivity for GIST [34]. Nevertheless, the mutational analysis performed with NGS requires only a small amount of viable tumor tissue. Therefore, it may contribute to the confirmation of GIST diagnosis in biopsies with a low cellular count. In one case of the present study, a gastric GIST, the EUS-FNB sample was low in cellular count, which made the immunostaining for GIST-specific markers inconclusive and difficult to interpret. However, the cell count was still sufficient for NGS, which unveiled a PDGFRA exon 18 p. (D842V) mutation.

Second, the mutation profile impacts the prognosis in GIST. PDGFRA mutations are associated with a favorable prognostic risk [35]. Gastric GISTs with KIT exon 11 deletions have a more malignant course of disease compared with $K I T$ exon 11 single nucleotide substitutions [36, 37]. Thus, the early detection of such mutations would support both clinicians and patients with important prognostic information.

Third, the TKI therapy response of GISTs as assessed by CT scan [38] can sometimes be misleading, as certain tumors that in fact respond to therapy do not decrease in size, but only in tumor density [39]. Hence, in such cases, there is an obvious risk that the clinician decides on TKI discontinuation despite a true anti-tumoral effect. Mutational analysis of pretreatment tumor tissue, as performed in the present study, cannot replace the evaluation by CT scan. Nevertheless, the analysis may act as an attractive complement by supporting the clinician with valuable genomic information and thereby facilitating the decision on continued neoadjuvant therapy. 


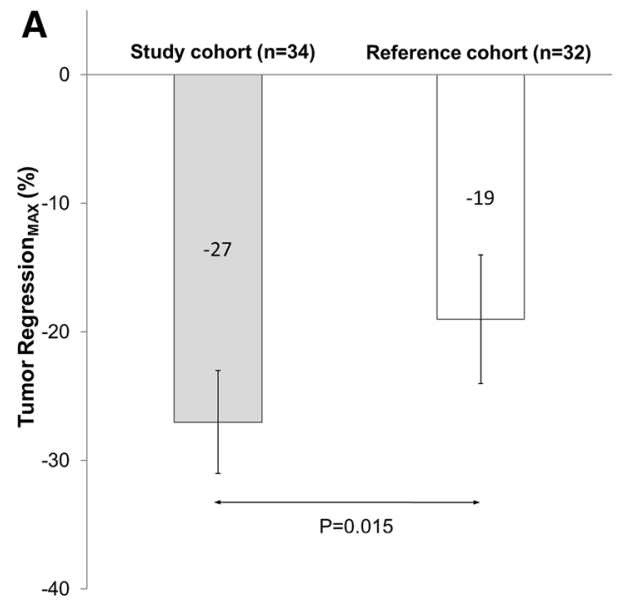

B $_{10}$

Study Cohort (SC)

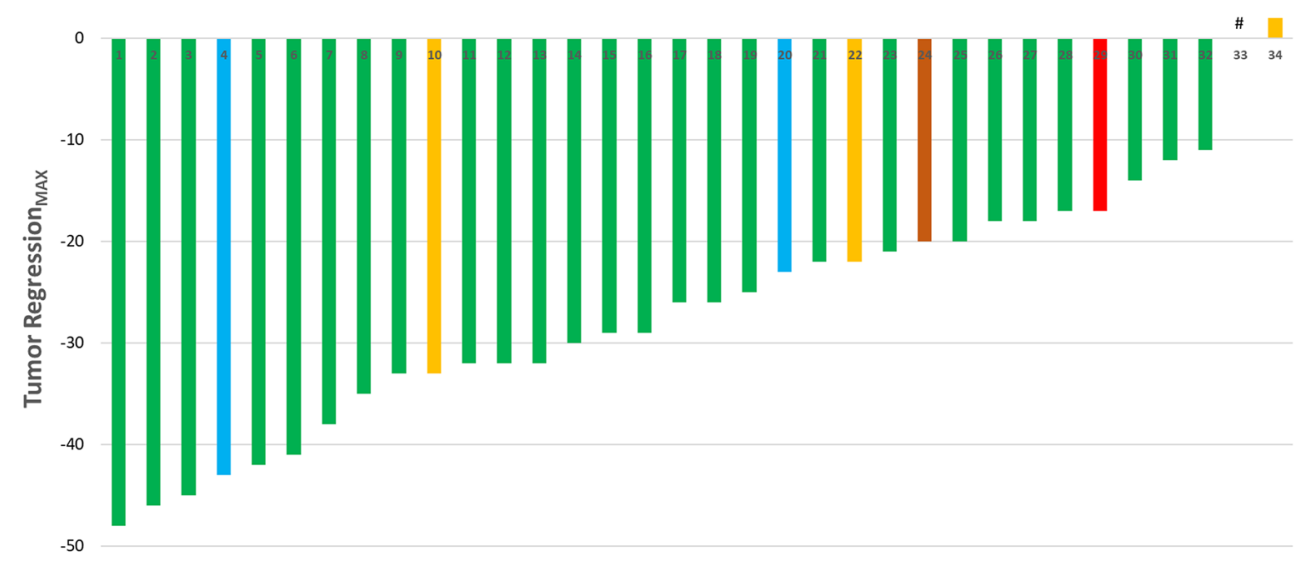

C

Reference Cohort (RC)

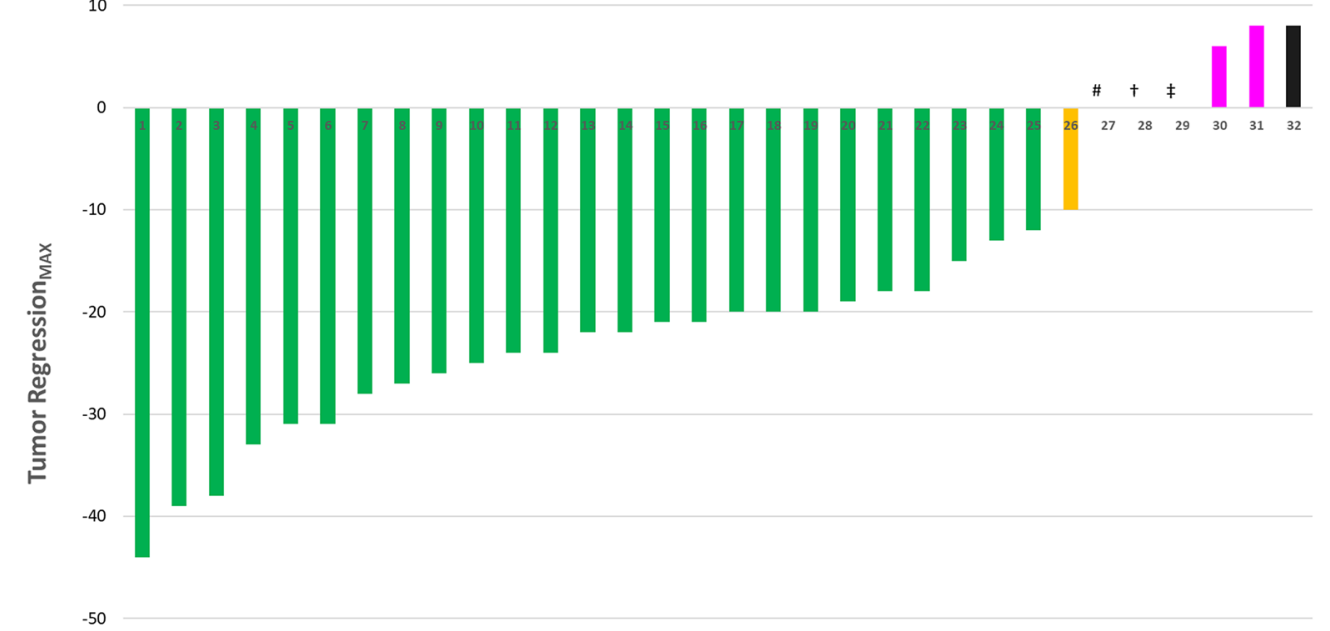


4Fig. 2 a Bar chart showing the preoperative tumor size reduction (Tumor Regression $_{\text {MAX) }}$ ) as evaluated by CT scan and induced by neoadjuvant TKI therapy in the study cohort (left bar in grey) and in the reference cohort (right bar in white). The error bars represent the $95 \%$ confidence interval. b Waterfall plot showing the preoperative tumor size reduction (Tumor Regression ${ }_{\mathrm{MAX}}$ ) in individual tumors of the study cohort (SC). The bar color represents the tumor genomic profile as follows: Bars in green: KIT exon 11-mutant (non p. L576P). Bars in blue: PDGFRA exon 18-mutant (non p. D842V). Bars in orange: KIT exon 13-mutant. Bars in brown: KIT exon 9-mutant. Bars in red: WT profile (case treated with neoadjuvant sunitinib and not imatinib). There was stable disease with neither tumor growth nor tumor regression in case number 33 (\#): KIT exon 9-mutant. c Waterfall plot showing the preoperative tumor size reduction (Tumor Regression $_{\mathrm{MAX}}$ ) in individual tumors of the reference cohort (RC). The bar color represents the tumor genomic profile as follows: Bars in green: KIT exon 11-mutant (non p. L576P). Bars in orange: KIT exon 13-mutant. Bars in purple: PDGFRA exon 18 p. (D842V)-mutant. Bars in black: KIT exon 11 p. (L576P)-mutant. There was stable disease with neither tumor growth nor tumor regression in case number 27 (\#): PDGFRA exon 18 p. (D842V)-mutant; case number $28(\dagger)$ : KIT exon 11 p. (L576P)-mutant; case number 29 (\$): PDGFRA exon 18 p. (D842V)-mutant

If available, the use of positron emission tomography-computed tomography (PET-CT) may also add information on the response to TKI therapy. In other studies, radiographic response on CT scan alone has been used to detect imatinib dose escalation [30] or imatinib discontinuation [10]. We believe that pretreatment mutational analysis in GISTs, at least in selected cases, should be considered in addition to the evaluation by CT scan.

The accurate pretreatment genetic profiling of GISTs is a multistep procedure dependent on an adequate sampling method, a dedicated pathologist, and a reliable sequencing method. Regarding the acquisition of the sample as such, EUS-FNB was highly accurate for GIST diagnosis in the present study, with the need for a repeated EUS-FNB only in one single case. Furthermore, all but a few tumors were indeed situated in locations within reach for oral access EUS. Therefore, the suggested work-up strategy with first priority EUS would be feasible in most large volume GIST centers.

As an alternative to EUS, we have shown that sequencing tissue acquired by the transabdominal approach (TUS-NB) is feasible in the few tumors situated in a position beyond the reach of EUS, e.g., distal small bowel GISTs. The TUS-NB procedure is safe [40]. The obvious drawback of TUS-NB is that some GISTs are small and located in deep gastric positions, making the transabdominal access demanding.

In 2016, we converted from Sanger sequencing to NGS. In the current study, we have shown that both techniques are reliable and can detect the mutations in GISTs with a high sensitivity. The advantage of NGS is that the technique requires a significantly lower amount of tumor DNA compared with the Sanger method and that it enables the simultaneous sequencing of multiple samples from different malignancies [41]. Consequently, the widespread clinical implementation of pretreatment NGS in GISTs would most likely reduce the mean time both to the completed analysis and to the initiation of genotype-driven neoadjuvant therapy.

GISTs that may have a WT profile constitute a hazardous subgroup of tumors as there might actually be a mutation. The frequency of WT tumors reported has gradually decreased [42], most likely due to improved methods of sequencing. In the $\mathrm{SC}$, we recorded only seven tumors (five resected, two nonresected) with a WT profile in the pretreatment sample. Post-treatment sequencing of the available five surgical specimens confirmed that three cases were true WT tumors, while in two cases, a primary mutation was detected-in both cases, a KIT exon 11 mutation. However, that potential mutations indeed exist in genes not included in the NGS panel cannot be excluded (Supplementary Table 3). Such an example is the potential existence of alterations in the SDH subunit. In the present study, the SDH status was not tested for by immunohistochemistry or by tumor genome sequencing. In addition, the low cellular count in a highly regressive resection specimen may compromise the mutational analysis and result in a false WT profile despite a true underlying mutation. Nevertheless, we believe that repeated sequencing of the corresponding surgical specimens is advisable in all cases where pretreatment sequencing has demonstrated a WT profile.

The criteria to be used to recommend neoadjuvant treatment in GIST remain to be determined [43, 44]. Treatment decisions are dependent on numerous factors, such as tumor size, tumor position, the existence of metastases, and the performance status of the patient. Unfortunately, there are few studies published on the topic. In the study by Rutkowski et al., patients with locally advanced tumors (no size criteria specified) [11] were given neoadjuvant therapy, while in the study by Kurokawa et al., tumors $>10 \mathrm{~cm}$ [10] were treated. As previously mentioned, in the current study, and according to the consensus at our tertiary center, more liberal criteria for neoadjuvant treatment are applied. This fact suggests that the presented results must be carefully interpreted and they might not be completely generalizable, especially in institutions that apply stricter criteria for neoadjuvant therapy.

An obvious drawback of neoadjuvant therapy in GISTs is that there is a risk for underestimation of the tumor prognostic risk (according to the NIH consensus criteria) in surgical specimens affected by long-term tyrosine kinase inhibition leading to tumor shrinkage and a decrease of the tumor proliferation rate (mitotic index) [45]. However, the Ki-67 index correlates relatively well to the mitotic rate and can potentially be used as a surrogate marker for the tumor proliferation rate [46, 47]. Consequently, in the current study, the decision on adjuvant therapy was based upon not only the surgical specimen analysis but also pretreatment data on tumor size at imaging and the Ki-67 index of EUS-FNB. 
Table 5 Short-term parameters related to surgery of GIST

\begin{tabular}{|c|c|c|c|}
\hline All tumors $(n=57)$ & Study cohort $(n=29)$ & Reference cohort $(n=28)$ & $P$ value \\
\hline \multicolumn{4}{|l|}{ Surgical procedure performed } \\
\hline Gastric wedge resection & 15 & 17 & \\
\hline Gastric sleeve resection & 2 & 0 & \\
\hline Gastrectomy (Billroth II) & 4 & 1 & \\
\hline Whipple resection & 3 & 2 & \\
\hline Small bowel resection & 2 & 4 & \\
\hline Extensive, multiorgan resection & 3 & 4 & \\
\hline \multicolumn{4}{|l|}{ Post-operative complications } \\
\hline No post-operative complication & 26 & 21 & 0.14 \\
\hline Yes, post-operative complication & 2 & 7 & \\
\hline Repeated surgery or endoscopic intervention required & $2^{\mathrm{a}}$ & $4^{\mathrm{b}}$ & \\
\hline Antibiotics required due to infection & 0 & 3 & \\
\hline \multicolumn{4}{|l|}{ Post-operative care } \\
\hline Duration of hospitalization (days), mean (SD) & $11.0(7.8)$ & $13.3(8.0)$ & 0.28 \\
\hline Tumors $<5 \mathrm{~cm}(n=21)$ & Study cohort $(n=12)$ & Reference cohort $(n=9)$ & $P$ value \\
\hline \multicolumn{4}{|l|}{ Surgical procedure performed } \\
\hline Gastric wedge resection & 7 & 8 & \\
\hline Gastric sleeve resection & 1 & 0 & \\
\hline Gastrectomy Billroth II & 3 & 0 & \\
\hline Whipple resection & 1 & 1 & \\
\hline Small bowel resection & 0 & 0 & \\
\hline Extensive, multiorgan resection & 0 & 0 & \\
\hline \multicolumn{4}{|l|}{ Post-operative complications } \\
\hline No post-operative complication & 11 & 7 & 0.12 \\
\hline Yes, post-operative complication & 1 & 4 & \\
\hline Repeated surgery or endoscopic intervention required & $1^{\mathrm{c}}$ & $2^{\mathrm{d}}$ & \\
\hline Antibiotics required due to infection & 0 & 2 & \\
\hline \multicolumn{4}{|l|}{ Post-operative care } \\
\hline Duration of hospitalization (days), mean (SD) & $12.1(9.9)$ & 14.2 (9.6) & 0.63 \\
\hline
\end{tabular}

GIST gastrointestinal stromal tumor, $S D$ standard deviation

${ }^{\text {a }}$ Leakage at the enteroanastomosis $(n=1)$; pyloric stenosis (endoscopic dilatation needed) $(n=1)$

${ }^{\mathrm{b}}$ Leakage at the anastomosis with the development of an abscess $(n=1)$; ileus $(n=1)$; leakage at the biliary anastomosis $(n=1)$; pyloric stenosis (endoscopic dilatation needed) $(n=1)$

${ }^{c}$ Pyloric stenosis (endoscopic dilatation needed) $(n=1)$

${ }^{\mathrm{d}}$ Leakage at the biliary anastomosis $(n=1)$; pyloric stenosis (endoscopic dilatation needed) $(n=1)$

This prospective study is strengthened by the fact that consecutive GIST patients of an entire, large region were included over a long period of time. Our center was responsible for the complete work-up process, including all clinical examinations and laboratory analyses, which ensured a standardized methodology. To the best of our knowledge, this is the first study to show the clinical and therapeutic benefits of pretreatment mutational analysis in GISTs in the setting of neoadjuvant TKI therapy.

There are some weaknesses in the presented study. For various reasons, such as discontinuation of therapy, not all study patients could be evaluated with CT scan after the initiation of imatinib therapy. The limited number of study patients did not allow us to draw any conclusions regarding the importance of pretreatment sequencing on the outcome of surgery after neoadjuvant therapy (R0 resection). Preferably, such a study should also be designed as a randomized, controlled trial thereby disqualifying some patients from pretreatment sequencing, which could be somewhat questionable from an ethical point of view.

We conclude that the performance of high-priority EUSFNB followed by immediate DNA sequencing of KIT and 
PDGFRA in the acquired pretreatment tumor tissue is highly valuable to guide neoadjuvant therapy in GISTs. Therefore, ineffective treatment with standard dose imatinib in unfavorable mutants, such as the PDGFRA exon $18 \mathrm{p} .(\mathrm{D} 842 \mathrm{~V})$ variant, can be minimized. The suggested approach is recommended to all centers responsible for the diagnostic workup and early care of patients with GISTs.

Acknowledgements Open access funding provided by University of Gothenburg. All staff at the GEA Endoscopy unit, Sahlgrenska University Hospital.

\section{Compliance with Ethical Standards}

Funding This work was not supported by any commercial or financial interests. Research grants were obtained from the following noncommercial foundations: The Health and Medical Care Committee of the Regional Executive Board, Region Västra Götaland (Grant number: VGFOUREG-564381 and VGFOUREG-144591); Sahlgrenska University Hospital (LUA-ALF, Grant number: 73830, 74370, and 70150); The Assar Gabrielsson Foundation (Grant number: 17-20); and Magtarmfonden (Grant number: 79211).

Conflict of interest Per Hedenström, Carola Andersson, Henrik Sjövall, Ola Nilsson, Bengt Nilsson, and Riadh Sadik declare they have no conflicts to report. Fredrik Enlund reports lecture honorarium from Novartis, Astra Zeneca, Roche, Lilly, and Astellas.

Ethical approval and informed consent This study was approved by the Regional Ethical Review Board of Gothenburg. Written informed consent was obtained from all the study participants. The study was registered at ClinicalTrials.gov (NCT02360839).

Open Access This article is licensed under a Creative Commons Attribution-NonCommercial 4.0 International License, which permits any non-commercial use, sharing, adaptation, distribution and reproduction in any medium or format, as long as you give appropriate credit to the original author(s) and the source, provide a link to the Creative Commons licence, and indicate if changes were made. The images or other third party material in this article are included in the article's Creative Commons licence, unless indicated otherwise in a credit line to the material. If material is not included in the article's Creative Commons licence and your intended use is not permitted by statutory regulation or exceeds the permitted use, you will need to obtain permission directly from the copyright holder.To view a copy of this licence, visit http://creativecommons.org/licenses/by-nc/4.0/.

\section{References}

1. Demetri GD, et al. Efficacy and safety of imatinib mesylate in advanced gastrointestinal stromal tumors. $\mathrm{N}$ Engl J Med. 2002;347(7):472-80.

2. Joensuu H, et al. Effect of the tyrosine kinase inhibitor STI571 in a patient with a metastatic gastrointestinal stromal tumor. $\mathrm{N}$ Engl J Med. 2001;344(14):1052-6.

3. Verweij $\mathbf{J}$, et al. Progression-free survival in gastrointestinal stromal tumours with high-dose imatinib: randomised trial. Lancet. 2004;364(9440):1127-34.

4. Blanke CD, et al. Phase III randomized, intergroup trial assessing imatinib mesylate at two dose levels in patients with unresectable or metastatic gastrointestinal stromal tumors expressing the KIT receptor tyrosine kinase: S0033. J Clin Oncol. 2008;26(4):626-32.

5. Dematteo RP, et al. Adjuvant imatinib mesylate after resection of localised, primary gastrointestinal stromal tumour: a randomised, double-blind, placebo-controlled trial. Lancet. 2009;373(9669):1097-104.

6. Gastrointestinal Stromal Tumor Meta-Analysis Group (MetaGIST). Comparison of two doses of imatinib for the treatment of unresectable or metastatic gastrointestinal stromal tumors: a meta-analysis of 1640 patients. J Clin Oncol. 2010;28(7):1247-53.

7. McAuliffe JC, et al. A randomized, phase II study of preoperative plus postoperative imatinib in GIST: evidence of rapid radiographic response and temporal induction of tumor cell apoptosis. Ann Surg Oncol. 2009;16(4):910-9.

8. Sjolund K, et al. Downsizing treatment with tyrosine kinase inhibitors in patients with advanced gastrointestinal stromal tumors improved resectability. World J Surg. 2010;34(9):2090-7.

9. Andtbacka RH, et al. Surgical resection of gastrointestinal stromal tumors after treatment with imatinib. Ann Surg Oncol. 2007;14(1):14-24.

10. Kurokawa Y, et al. Phase II study of neoadjuvant imatinib in large gastrointestinal stromal tumours of the stomach. Br J Cancer. 2017;117(1):25-32.

11. Rutkowski $\mathrm{P}$, et al. Neoadjuvant imatinib in locally advanced gastrointestinal stromal tumors (GIST): the EORTC STBSG experience. Ann Surg Oncol. 2013;20(9):2937-43.

12. Wang D, et al. Phase II trial of neoadjuvant/adjuvant imatinib mesylate for advanced primary and metastatic/recurrent operable gastrointestinal stromal tumors: long-term follow-up results of Radiation Therapy Oncology Group 0132. Ann Surg Oncol. 2012;19(4):1074-80.

13. Lasota J, Miettinen M. Clinical significance of oncogenic KIT and PDGFRA mutations in gastrointestinal stromal tumours. Histopathology. 2008;53(3):245-66.

14. Heinrich $\mathrm{MC}$, et al. Kinase mutations and imatinib response in patients with metastatic gastrointestinal stromal tumor. J Clin Oncol. 2003;21(23):4342-9.

15. Debiec-Rychter M, et al. KIT mutations and dose selection for imatinib in patients with advanced gastrointestinal stromal tumours. Eur J Cancer. 2006;42(8):1093-103.

16. Corless CL, et al. PDGFRA mutations in gastrointestinal stromal tumors: frequency, spectrum and in vitro sensitivity to imatinib. J Clin Oncol. 2005;23(23):5357-64.

17. Conca E, et al. Activate and resist: L576P-KIT in GIST. Mol Cancer Ther. 2009;8(9):2491-5.

18. ESMO/European Sarcoma Network Working Group. Gastrointestinal stromal tumors: ESMO Clinical Practice Guidelines for diagnosis, treatment and follow-up. Ann Oncol. 2012;23(Suppl 7):vii49-55.

19. Hirota S, et al. Gain-of-function mutations of platelet-derived growth factor receptor alpha gene in gastrointestinal stromal tumors. Gastroenterology. 2003;125(3):660-7.

20. Nishida $T$, et al. The standard diagnosis, treatment, and follow-up of gastrointestinal stromal tumors based on guidelines. Gastric Cancer. 2016;19(1):3-14.

21. Yip D, et al. ALT-GIST: Randomized phase II trial of imatinib alternating with regorafenib versus imatinib alone for the firstline treatment of metastatic gastrointestinal stromal tumor (GIST). 2019;37(15_suppl):11023.

22. Agaimy A, et al. Value of epithelioid morphology and PDGFRA immunostaining pattern for prediction of PDGFRA mutated genotype in gastrointestinal stromal tumors (GISTs). Int J Clin Exp Pathol. 2013;6(9):1839-46. 
23. Hedenbro JL, Ekelund M, Wetterberg P. Endoscopic diagnosis of submucosal gastric lesions The results after routine endoscopy. Surg Endosc. 1991;5(1):20-3.

24. Watson RR, et al. Yield and performance characteristics of endoscopic ultrasound-guided fine needle aspiration for diagnosing upper GI tract stromal tumors. Dig Dis Sci. 2011;56(6):1757-62.

25. Hedenstrom $\mathrm{P}$, et al. Characterizing gastrointestinal stromal tumors and evaluating neoadjuvant imatinib by sequencing of endoscopic ultrasound-biopsies. World J Gastroenterol. 2017;23(32):5925-35.

26. Trotti A, et al. CTCAE v3.0: development of a comprehensive grading system for the adverse effects of cancer treatment. Semin Radiat Oncol. 2003;13(3):176-181.

27. Therasse $\mathrm{P}$, et al. New guidelines to evaluate the response to treatment in solid tumors. European Organization for Research and Treatment of Cancer, National Cancer Institute of the United States, National Cancer Institute of Canada. J Natl Cancer Inst. 2000;92(3):205-16.

28. Cassier PA, et al. Outcome of patients with platelet-derived growth factor receptor alpha-mutated gastrointestinal stromal tumors in the tyrosine kinase inhibitor era. Clin Cancer Res. 2012;18(16):4458-64.

29. Wang CM, et al. Secondary resistance to imatinib in patients with gastrointestinal stromal tumors through an acquired KIT exon 17 mutation. Mol Med Rep. 2009;2(3):455-60.

30. Doyon $\mathrm{C}$, et al. Prolonged therapy with imatinib mesylate before surgery for advanced gastrointestinal stromal tumor results of a phase II trial. Int J Surg Oncol. 2012;2012:761576.

31. Blesius A, et al. Neoadjuvant imatinib in patients with locally advanced non metastatic GIST in the prospective BFR14 trial. BMC Cancer. 2011;11:72.

32. Eisenberg BL, et al. Phase II trial of neoadjuvant/adjuvant imatinib mesylate (IM) for advanced primary and metastatic/recurrent operable gastrointestinal stromal tumor (GIST): early results of RTOG 0132/ACRIN 6665. J Surg Oncol. 2009;99(1):42-7.

33. Medeiros F, et al. KIT-negative gastrointestinal stromal tumors: proof of concept and therapeutic implications. Am J Surg Pathol. 2004;28(7):889-94.

34. Hwang DG, Qian X, Hornick JL. DOG1 antibody is a highly sensitive and specific marker for gastrointestinal stromal tumors in cytology cell blocks. Am J Clin Pathol. 2011;135(3):448-53.

35. Lasota J, et al. A great majority of GISTs with PDGFRA mutations represent gastric tumors of low or no malignant potential. Lab Invest. 2004;84(7):874-83.
36. Miettinen M, Sobin LH, Lasota J. Gastrointestinal stromal tumors of the stomach: a clinicopathologic, immunohistochemical, and molecular genetic study of 1765 cases with long-term follow-up. Am J Surg Pathol. 2005;29(1):52-68.

37. Nilsson B, et al. Adjuvant imatinib treatment improves recurrencefree survival in patients with high-risk gastrointestinal stromal tumours (GIST). Br J Cancer. 2007;96(11):1656-8.

38. Le Cesne A, et al. Absence of progression as assessed by response evaluation criteria in solid tumors predicts survival in advanced GI stromal tumors treated with imatinib mesylate: the intergroup EORTC-ISG-AGITG phase III trial. J Clin Oncol. 2009;27(24):3969-74.

39. Choi $\mathrm{H}$, et al. Correlation of computed tomography and positron emission tomography in patients with metastatic gastrointestinal stromal tumor treated at a single institution with imatinib mesylate: proposal of new computed tomography response criteria. J Clin Oncol. 2007;25(13):1753-9.

40. Eriksson M, et al. Needle biopsy through the abdominal wall for the diagnosis of gastrointestinal stromal tumour-does it increase the risk for tumour cell seeding and recurrence? Eur J Cancer. 2016;59:128-33.

41. Verma M, Kulshrestha S, Puri A. Genome sequencing. Methods Mol Biol. 2017;1525:3-33.

42. Patrikidou A, et al. Long-term outcome of molecular subgroups of GIST patients treated with standard-dose imatinib in the BFR14 trial of the French Sarcoma Group. Eur J Cancer. 2016;52:173-80.

43. Etherington MS, DeMatteo RP. Tailored management of primary gastrointestinal stromal tumors. Cancer. 2019;125:2164-71.

44. Ishikawa $\mathrm{T}$, et al. Neoadjuvant therapy for gastrointestinal stromal tumor. Transl Gastroenterol Hepatol. 2018;3:3.

45. Joensuu $\mathrm{H}$, et al. Risk of recurrence of gastrointestinal stromal tumour after surgery: an analysis of pooled population-based cohorts. Lancet Oncol. 2012;13(3):265-74.

46. Kemmerling R, et al. Robust linear regression model of Ki-67 for mitotic rate in gastrointestinal stromal tumors. Oncol Lett. 2014;7(3):745-9.

47. Zhou Y, et al. Ki67 is a biological marker of malignant risk of gastrointestinal stromal tumors: a systematic review and metaanalysis. Medicine (Baltimore). 2017;96(34):e7911.

48. Nishida T, Blay JY, Hirota S, et al. The standard diagnosis, treatment, and follow-up of gastrointestinal stromal tumors based on guidelines. Gastr Cancer. 2016;19(1):3-14. https://doi. org/10.1007/s10120-015-0526-8. 